Check for updates

Cite this: RSC Adv., 2019, 9, 35109

\title{
Direct biodiesel production from wet spent coffee grounds $\uparrow$
}

\begin{abstract}
Juliati Br. Tarigan, (D) *a Mimpin Ginting, (DD a Siti Nurul Mubarokah, ${ }^{\mathrm{b}}$ Firman Sebayang, ${ }^{\mathrm{a}}$ Justaman Karo-karo, ${ }^{c}$ Trung T. Nguyen, de Junedi Ginting ${ }^{f}$ and Eko K. Sitepu (DD ${ }^{a}$

Utilization of waste spent coffee grounds (SCG) remains limited and requires pre-treatment before being discarded to avoid pollution to the environment. Lipids contained in SCG could be converted to biodiesel through an in situ transesterification method. Current in situ transesterification of wet SCG biomass, conducted at high reaction temperature to reduce the water effect and reduce reaction time, is energy intensive. A new approach, which combines simultaneous extraction-transesterification in a single step using soxhlet apparatus, was developed to produce biodiesel directly from wet SCG biomass. A homogeneous base catalyst at a concentration of $0.75 \mathrm{M}$ showed better catalytic activity than acid, with hexane as a co-solvent on fatty acid (FA) extraction efficiency and FA to fatty acid methyl ester (FAME) conversion efficiency. Studying the factorial effect of ratio of methanol to hexane and reaction time led to the highest FA to FAME conversion efficiency of $97 \%$ at a ratio of $1: 2$ and 30 min reaction time. In addition, the catalyst could be used five times without losing its activity. In term of energy consumption, the reactive extraction soxhlet (RES) method could save 38-99\% of energy compared to existing methods.
\end{abstract}

Received 3rd October 2019
Accepted 25th October 2019

DOI: $10.1039 / c 9 r a 08038 d$

rsc.li/rsc-advances

transesterification of oilseed to biodiesel could reduce total

\section{Introduction}

Demand on renewable energy has increased rapidly as a consequence of the decreasing non-renewable fossil fuel reserve and rising environmental concerns. ${ }^{1}$ Among liquid renewable energy, biodiesel has been identified as able to be used directly in a diesel engine without requiring any modification. ${ }^{2}$ Unlike vegetable oils, which have high viscosity, low cetane number and low flash point, biodiesel offers the opposite properties which are more suitable for modern diesel engines. ${ }^{3}$ However, current multiple-step biodiesel production requires preparation of oil/lipid as raw material, which is very costly. Economic model analysis by Haas et al. ${ }^{4}$ predicted that $88 \%$ of total biodiesel production cost accounted for oil feedstocks. Therefore eliminating extraction and purification of oil through in situ

${ }^{a}$ Department of Chemistry, Faculty of Mathematics and Natural Sciences, Universitas Sumatera Utara, Medan 20155, Indonesia. E-mail: juliati@usu.ac.id

${ }^{b}$ Medical Faculty, University of Islam Malang, Malang 65144, Indonesia

${ }^{\circ}$ Balai Riset dan Standarisasi Industri, Kementerian Perindustrian, Medan 20214, Indonesia

${ }^{d}$ Department of Food Technology, An Giang University, Long Xuyen City, Vietnam ${ }^{e}$ Department of Medical Biotechnology, Flinders University, Bedford Park 5043, Adelaid, South Australia

${ }^{f}$ Department of Physics, Faculty of Mathematics and Natural Sciences, Universitas Sumatera Utara, Medan 20155, Indonesia

$\dagger$ Electronic supplementary information (ESI) available. See DOI: $10.1039 / \mathrm{c} 9 \mathrm{ra} 08038 \mathrm{~d}$ biodiesel production cost. ${ }^{5,6}$

Some researchers have confirmed that a high biodiesel yield could be produced from in situ transesterification of crop seed/ biomass. $^{7-11}$ Furthermore, Dasari et al. ${ }^{12}$ confirmed that biodiesel from the in situ transesterification method meets the ASTM standard and can be used to power the diesel engine. Currently biodiesel production relies on vegetable oil as a source, which further exacerbates the food $v s$. fuel debate. ${ }^{13}$ The utilization of feedstocks which require a lot of water for cultivation (drink vs. fuel) has also been critiqued. ${ }^{\mathbf{1 4}}$ Consequently finding low-cost feedstocks which contain high lipids is necessary to increase biodiesel competitiveness. Despite waste cooking oil price is 2 to 3 times lower than vegetable oil, ${ }^{15}$ the supplies are limited and contain high impurities. ${ }^{\mathbf{1 6}}$

Waste biomass, for example spent coffee grounds (SCG), has been identified as containing lipids in the range of 10$28 \% .{ }^{17,18}$ Utilization of SCG remains limited, such as for embankment fill material, ${ }^{\mathbf{1 9}}$ composting, and mushroom cultivation media. ${ }^{20}$ Nescafe, the largest instant coffee producer, has used SCG as a fuel for their boilers in some factories. Furthermore, SCG require waste treatment before it can be discarded, as it contains acid which could contaminate the environment. ${ }^{21,22}$ The amount of SCG has increased every year as coffee is the favourite beverage in the world with 9.5 million ton consumed in 2016, ${ }^{23}$ of which half was instant coffee. ${ }^{17}$ Each gram of coffee could produce $0.65-0.91$ gram of SCG depending on the brewing method and coffee species. ${ }^{24,25}$ 
Hence, a proper waste management system is required to avoid environmental impact.

In situ transesterification of SCG is the most economical method to valorize waste SCG biomass through direct extraction and conversion of valuable oil to biodiesel. Tuntiwiwattanapun et $a{ }^{17}{ }^{17}$ demonstrated the direct biodiesel production from dry SCG using $\mathrm{NaOH}$ as catalyst, which generated $77 \%$ biodiesel yield in $3 \mathrm{~h}$ reaction time at $50{ }^{\circ} \mathrm{C}$. Scale up of the process was conducted to produce biodiesel which meets the ASTM standard. ${ }^{17}$ In order to make the process more efficient, isopropanol as a cosolvent was used and could improve biodiesel yield to $89 \%$ in $2 \mathrm{~h}$ reaction time. ${ }^{26}$ However, those studies used dry SCG biomass which requires a drying stage. The dewatering process is an energy penalty. For example, $89 \%$ of total energy input was accounted for by the dewatering microalgae biomass. ${ }^{27}$ Alternatively, in situ transesterification of wet SCG has been conducted with and without catalyst supported by co-solvent. Even though satisfactory biodiesel yields occurred from those experiments, the in situ transesterification process involved high reaction temperature $\left(95-270{ }^{\circ} \mathrm{C}\right.$ ) in a prolonged reaction time (20 min to $3 \mathrm{~h}$ ), which increased the total biodiesel production cost. ${ }^{21,28,29}$

Several studies have shown that simultaneous extraction and transesterification in different chambers in one system could produce biodiesel directly from biomass with a high yield. ${ }^{30-32}$ Continuous contact of fresh solvent with biomass facilitates the lipid transfer which is further converted to biodiesel with a catalyst. In addition, Liu et al. ${ }^{25}$ successfully impregnated the dry SCG biomass with $\mathrm{H}_{2} \mathrm{SO}_{4}$ and the simultaneous extractiontransesterification reaction was processed in the extraction chamber with methanol. However, a total $39 \mathrm{~h}$ reaction time was required for the impregnation and in situ transesterification process, which is cost and energy intensive. Therefore, this present study aimed to produce biodiesel directly from wet SCG biomass using the reactive extraction soxhlet (RES) method and hexane as co-solvent. The single effect of different types of homogeneous catalysts and it catalytic efficiency, co-solvents and catalyst concentration was determined while interaction between reaction time and ratio of biomass to co-solvent was established in factorial design. In addition the reusability of the catalyst was also determined. Finally, the energy consumption of RES method was determined and compared with other in situ transesterification and two-step using either dry or wet SCG.

\section{Experimental}

\subsection{Materials}

SCG biomass was collected from the local coffee shop in Medan, Sumatera Utara - Indonesia and stored in a cold room before use. All chemicals used in this study were purchased from a local chemicals dealer and were used without any treatment.

\subsection{Conventional in situ transesterification of wet SCG}

\section{biomass}

In situ transesterification of wet SCG biomass using the conventional reflux method was used as a control experiment. Homogeneous sulphuric acid or sodium hydroxide with a concentration of $0.75 \mathrm{M}$ was used as catalysts. Briefly, 10 gram of wet SCG was mixed with $75 \mathrm{~mL}$ of methanol containing the catalyst and $75 \mathrm{~mL}$ hexane as co-solvent in a $250 \mathrm{~mL}$ round bottom flask equipped with condenser. The reaction was conducted for $30 \mathrm{~min}$ and the hexane containing product was separated and washed 3 times with $25 \mathrm{~mL}$ aquadest. Next, the hexane phase was dried under a nitrogen stream and reconstituted with chloroform-D for ${ }^{1} \mathrm{H}$-NMR analysis.

\subsection{In situ transesterification of wet SCG biomass using the RES}

Direct biodiesel production from wet SCG biomass was conducted in a soxhlet apparatus that has two separate chambers. 10 gram of wet SCG biomass in a thimble was placed in the extraction chamber of the soxhlet connected to the condenser and $150 \mathrm{~mL}$ mixture of methanol containing the catalyst and hexane with investigated ratio (v/v) was poured into reaction chamber. Reaction time started when the co-solvent in the extraction chamber dropped down into the reaction chamber and continued based on investigated time. Different catalyst types (homogeneous acid and base), catalyst concentrations, cosolvents, and ratios of methanol to co-solvent were explored to determine the optimum condition for an in situ transesterification process. The biodiesel dissolved in hexane was separated and washed 3 times with $25 \mathrm{~mL}$ aquadest to remove impurities. After evaporating the hexane phase, the biodiesel was reconstituted with chloroform-D for ${ }^{1} \mathrm{H}$-NMR analysis. Standard errors were calculated based on triplicate experiments.

\subsection{Lipid determination}

Lipid content in SCG was determined using the Folch method with slight modification. ${ }^{33}$ Firstly, $50 \mathrm{mg}$ of dry SCG was vortexed with $1.4 \mathrm{~mL}$ saline water and $2 \mathrm{~mL}$ methanol. Next, $4 \mathrm{~mL}$ chloroform was added and vortexed again for $5 \mathrm{~min}$. The chloroform phase was collected and stored. The addition of $4 \mathrm{~mL}$ chloroform was repeated 2 times more to make sure all lipids have been extracted from SCG. The chloroform layer was then evaporated under a nitrogen stream and the total lipids were determined gravimetrically.

\subsection{Fatty acid methyl ester profile}

The fatty acid profile of SCG was determined using gas chromatography using a mass spectrophotometer (GC-MS). A $1 \mu \mathrm{L}$ of fatty acid methyl ester (FAME) derived from SCG was injected to GC-MS (Agilent technology 5975C) equipped with a capillary column (length $15 \mathrm{~m}$ ID $0.25 \mathrm{~mm}$ ) and a flame ionization detector. Helium gas was employed as the carrier gas with constant delivered flow of $1 \mathrm{~mL} \mathrm{~min}^{-1}$. The split ratio of $1: 20$ was used and the injection port temperature was set at $240{ }^{\circ} \mathrm{C}$ while detector temperature was maintained at $250{ }^{\circ} \mathrm{C}$.

\subsection{Biodiesel confirmation and yield}

Confirmation and determination of biodiesel yield were performed using FT-IR (PerkinElmer, FT-IR 100) and ${ }^{1} \mathrm{H}-\mathrm{NMR}(600$ $\mathrm{MHz}$ Bruker spectrophotometer) as this is a rapid and reliable 
method used in the biodiesel industry. ${ }^{34}$ The FA to FAME conversion efficiency was calculated based on the integration value of specific chemical shift of methylene and methoxy proton generated by spectroscopy ${ }^{1} \mathrm{H}$-NMR. The ${ }^{1} \mathrm{H}-\mathrm{NMR}$ analysis was set up on 64 scans and 1 s D1 delay. The FA extraction efficiencies were calculated based on the equations developed by previous researchers. ${ }^{35}$

\section{Results and discussion}

The oil extracted from SCG biomass was $12 \%$ of its dry matter, which is in the range of oil content in SCG. ${ }^{17,18}$ The water content in SCG was $61 \%$, which is similar to other researchers findings. ${ }^{21,29}$ The saponifiable compounds of SCG composed of free fatty acid $(0.7 \%)$, monoglycerides $(0.14 \%)$, diglycerides (6.68\%) and triglycerides (92.48\%), while no unsaponifiable compound was detected. The fatty acid profile of SCG biomass, as shown in Table 1, was determined using GC/MS dominated by palmitic acid (32.9\%) and linoleic acid (44.1\%). The total saturated fatty acid was $39.6 \%$, monounsaturated fatty acid was $9.4 \%$ and polyunsaturated fatty acid was $44 \%$.

The common lipid extraction method using a soxhlet apparatus was adopted to produce biodiesel directly from wet SCG biomass. The soxhlet apparatus consists of two separate chambers with one chamber an extraction zone and the other the reaction zone. In this apparatus water contained in wet SCG biomass cannot inhibit the transesterification reaction as the non-polar solvent carries only the lipids to the reaction zone. Choosing the starting extraction-transesterification processing time is crucial, as it has to ensure that all the lipids have been extracted and converted to biodiesel. Al-Hamamre et al. ${ }^{36}$ demonstrated that the highest coffee oil yield occurred after 30 min extraction of SCG biomass using hexane as a solvent. Furthermore, Uzun et al. ${ }^{37}$ confirmed that $30 \mathrm{~min}$ reaction time was enough to convert lipid to biodiesel. Therefore the minimum reaction time used in this study was set to $30 \mathrm{~min}$.

The published equations based on ${ }^{1} \mathrm{H}$-NMR spectra ${ }^{35}$ were used to calculate the FA extraction efficiencies derived from in

Table 1 Fatty acid profile of SCG biomass

\begin{tabular}{llc}
\hline Fatty Acid & & \\
\hline $\mathrm{C}_{12: 0}$ & Lauric & Percentage \\
$\mathrm{C}_{14: 0}$ & Myristic & 0.5 \\
$\mathrm{C}_{16: 0}$ & Palmitic & 0.3 \\
$\mathrm{C}_{17: 0}$ & Margaric & 32.9 \\
$\mathrm{C}_{18: 0}$ & Stearic & 0.1 \\
$\mathrm{C}_{20: 0}$ & Arachidic & 7.5 \\
$\mathrm{C}_{22: 0}$ & Behenic & 2.8 \\
$\mathrm{C}_{24: 0}$ & Lignoceric & 0.6 \\
$\mathrm{C}_{18: 1}$ trans & Oleic & 0.2 \\
$\mathrm{C}_{18: 1}$ cis & Oleic & 0.04 \\
$\mathrm{C}_{18: 2}$ trans & Linoleic & 9.02 \\
$\mathrm{C}_{18: 2}$ cis & Linoleic & 0.08 \\
$\mathrm{C}_{18: 3}$ trans & Linolenic & 44.1 \\
$\mathrm{C}_{18: 3}$ cis & Linolenic & 0.02 \\
$\mathrm{C}_{20: 1}$ & Gondoic & 1.4 \\
$\mathrm{C}_{22: 1}$ & Erucic & 0.3 \\
& & 0.05
\end{tabular}

situ transesterification of wet SCG using RES method and were compared with FA profiles determined by GC-MS analysis using the Folch method. The FA extraction efficiencies occurred on average of $58.1 \mathrm{~mol} \%$ ranging from $48.6-78.1 \mathrm{~mol} \%$. Linolenic acid was not quantifiable due to low concentration in SCG $(1.3 \%)$ while palmitic and stearic acids were not extracted sufficiently.

\subsection{Effect of catalyst type}

Homogeneous catalysts have been identified as having better catalytic activity in the transesterification reaction than heterogeneous catalysts and enzymes. ${ }^{38}$ Homogeneous acid and base catalysts, such as sulphuric acid and sodium hydroxide, were used to determine the effect of catalyst type in this study. The concentration of both catalysts was set to $0.75 \mathrm{M}$ in methanol while the ratio of methanol to hexane was $1: 1$. The FA to FAME conversion efficiencies were determined based on different reaction times, from $30 \mathrm{~min}$ to $90 \mathrm{~min}$ with $15 \mathrm{~min}$ increments. As predicted, the homogeneous base catalyst showed superior catalytic activity against the homogenous acid catalyst (Fig. 1A). The FA to FAME conversion efficiencies obtained from sodium hydroxide as catalyst routinely occurred more than $90 \%$ in all reaction times, while sulphuric acid showed low conversion efficiencies, with the highest occurring after $90 \mathrm{~min}$ reaction time $(4.6 \%)$. This result supports the previously published result that a homogeneous base catalyst could catalyze biodiesel formation faster. ${ }^{6,39}$ It worth noting that water contained in wet SCG biomass did not deter transesterification reaction even though some of the water dropped down into the reaction chamber together with the methanol and evaporated to the extraction chamber. This is because the lipids extracted from wet SCG biomass were dissolved in hexane which has different polarity with water.

Both acid and base catalysts did not have a significant effect on the extraction efficiencies of $\mathrm{C}_{18: 2}$ and $\mathrm{C}_{18: 1}$ (Fig. 2A). The base catalyst could extract $\mathrm{FA}$ better than the acid catalyst with average of 67.2 and $50.26 \mathrm{~mol} \%$, respectively. However, the acid catalyst showed a more stable extraction efficiency of $\mathrm{C}_{18: 2}$ at \pm $21.2 \mathrm{~mol} \%$ with increasing reaction time than the base catalyst, with results of FA extraction efficiency ranging from $10 \pm 3.5$ to $17.5 \pm 0.7 \mathrm{~mol} \%$. This result is in contrast with previous published extraction efficiencies obtained using vortex fluidic device intensified direct transesterification of wet microalgae Chloroparva pannonica biomass which concluded that the acid catalyst has higher FA extraction efficiencies on $\mathrm{C}_{16: 0}$ and $\mathrm{C}_{18: 3}$ at 85 and $91 \mathrm{~mol} \%$ than 54 and 49 mol\% using the base catalyst. ${ }^{6}$

\subsection{Effect of co-solvent}

Some studies of the in situ transesterification process have demonstrated that the co-solvent can enhance oil extraction and reduce methanol usage, in particular non-polar solvents which can dissolve triglycerides. ${ }^{5,40}$ In this study, as the in situ transesterification was conducted in a soxhlet apparatus, the effect of different co-solvents was examined using chloroform, hexane, and acetone. The experiments were set to operate using the ratio of methanol to co-solvent of $1: 1$, with $0.75 \mathrm{M}$ sodium 

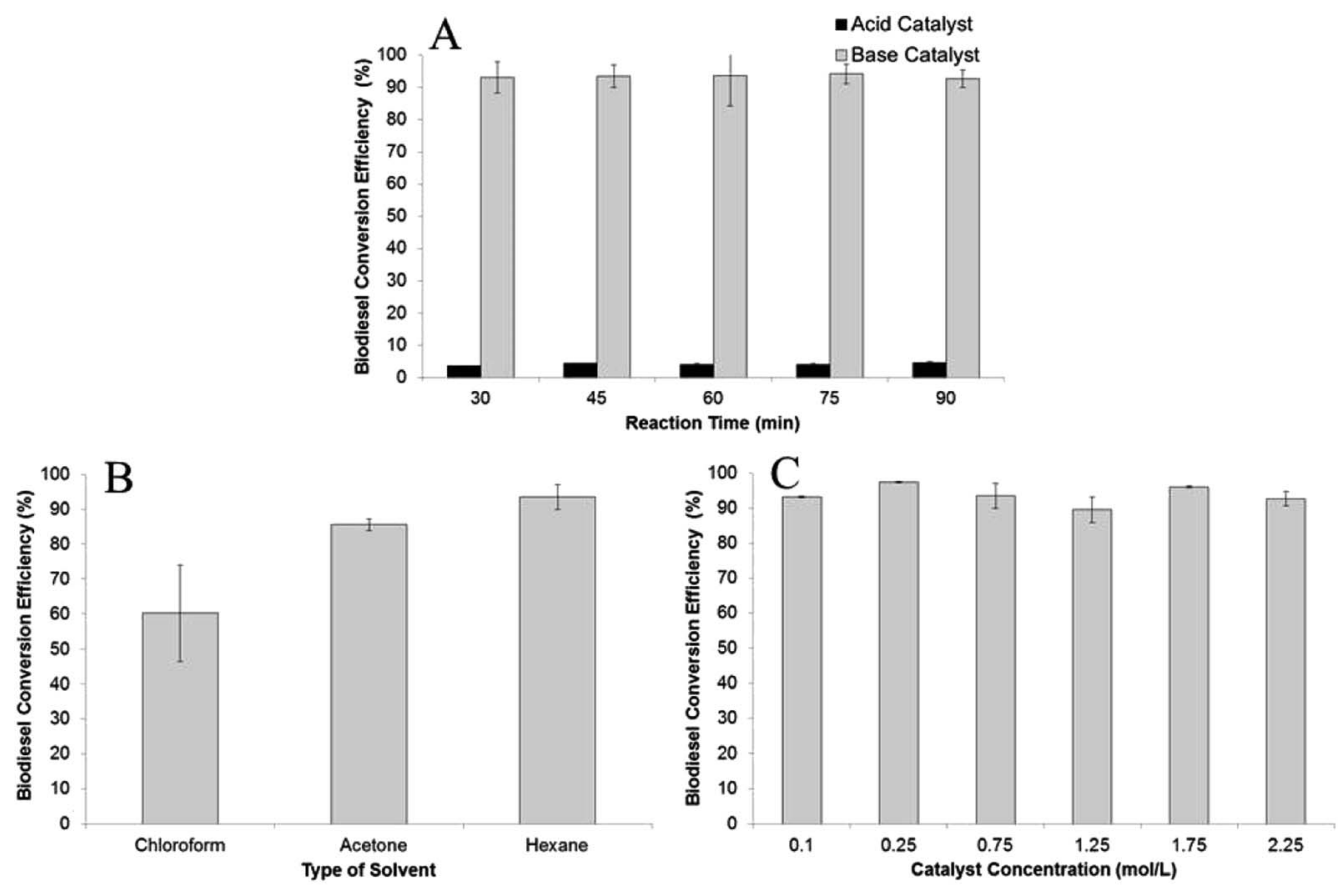

Fig. 1 The effect of (A) catalyst type; (B) type of solvent; and (C) catalyst concentration ( $\mathrm{NaOH}$ ) on biodiesel conversion efficiency.

hydroxide in methanol as the catalyst (Fig. 1B). A control experiment using methanol without the addition of the cosolvent resulted in no FA to FAME detection after $30 \mathrm{~min}$ reaction time. The explanation for this result could be that the solubility of triglycerides in methanol is very $\operatorname{low}^{7}$ and therefore no lipids transferred to reaction chamber. In contrast, acetone, which dissolves in methanol forming a homogeneous mixture, showed an FA to FAME conversion efficiency of $86 \%$. This result was lower than with the use hexane (94\%) because some water from the wet biomass was extracted by the acetone generating saponification. The white semi-solid soap appeared at the end of the processing time. The lowest FA to FAME conversion efficiency of $60 \%$ was noticed using chloroform as co-solvent. Again, soap formation lowered the concentration of the catalyst in reactants, as the chloroform caused water to drop down into the reaction chamber. As shown in Fig. 2B, hexane had higher FA extraction efficiencies for both $\mathrm{C}_{18: 2}$ and $\mathrm{C}_{18: 1}$ than chloroform and acetone. Hexane is a non-polar solvent which dissolved the lipids from the wet SCG and caused them to react with methanol with the addition of the catalyst.
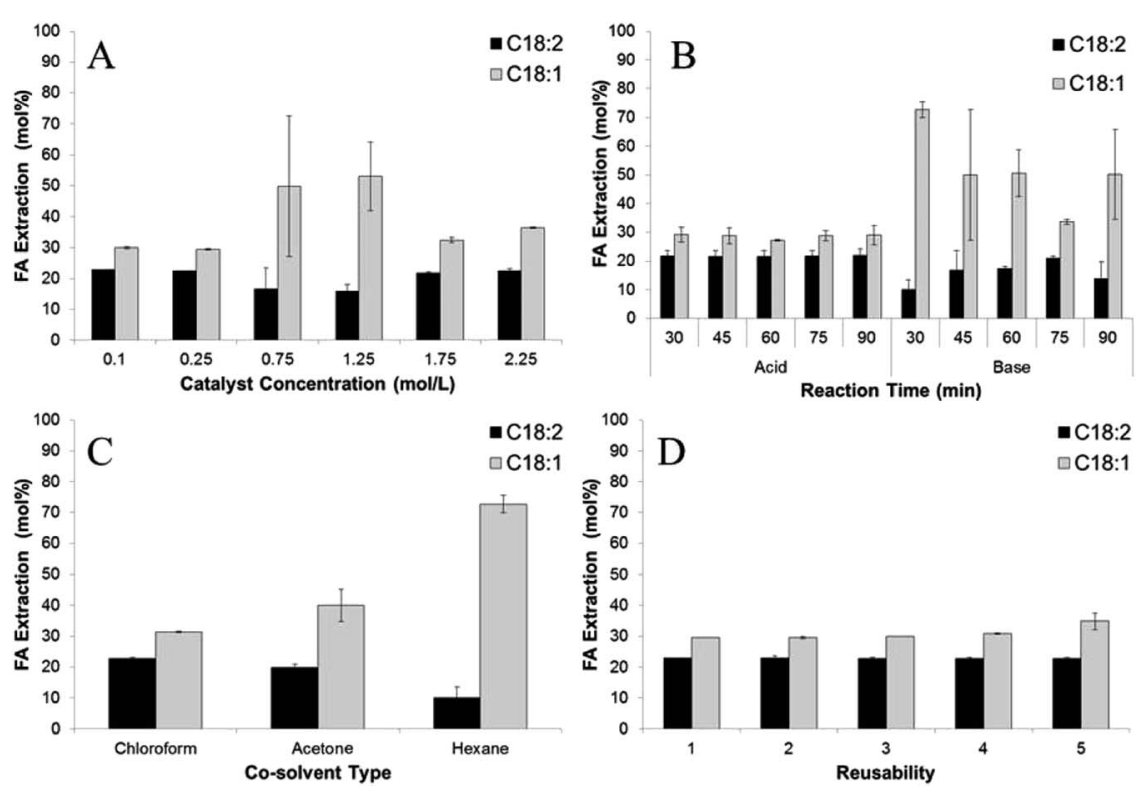

Fig. 2 The effect of (A) catalyst concentration; (B) type of catalyst; (C) co-solvent type; and (D) reusability of the catalyst on FA extraction. 


\subsection{Effect of catalyst concentration}

As mentioned above, the homogeneous base catalyst has bigger catalytic activity than acid on conversion efficiency, which we therefore used in the present study to determine the effect of catalyst concentration. This parameter was investigated using various concentrations from $0.1 \mathrm{M}$ to $2.25 \mathrm{M}$ and a $1: 1$ of ratio of methanol to hexane for $45 \mathrm{~min}$ reaction time. As recorded, the conversion efficiency of $\geq 90 \%$ occurred for all catalyst concentrations used, with the highest (97\%) obtained using $0.25 \mathrm{M}$ sodium hydroxide in methanol (Fig. 1C). This result is similar to other published results of in situ transesterification of microalgae and fungal biomass, which showed that $>1 \%$ catalyst concentration achieving $\geq 90 \%$ conversion efficiency. ${ }^{\mathbf{1 0}, \mathbf{1 1}}$ Catalyst concentration had no significant effect on $\mathrm{C}_{18: 2}$ extractions with average extraction efficiency of $20.4 \mathrm{~mol} \%$, as shown in Fig. 2C. Similarly, no significant effect was achieved on extraction efficiency of $\mathrm{C}_{18: 1}$ with efficiencies ranging from $29.4 \pm 0.5-53.1 \pm 11.1$.

\subsection{Effect of the ratio of methanol to co-solvent}

Based on the effect of the co-solvent study, hexane was used to extract lipids and bring them to the reaction chamber for the transesterification process. The ratio of methanol to hexane was critical, as it affects the amount of triglyceride extracted, which governs the biodiesel yield. Therefore the effect of the ratio of methanol to hexane was investigated at five different ratios using $0.75 \mathrm{M}$ sodium hydroxide in methanol as catalyst at five different reaction time-examined from $30 \mathrm{~min}$ to $90 \mathrm{~min}$. As shown in Fig. 3, the methanol to hexane ratios of $1: 0.5$ to $1: 3$ had no significant effect on FA to FAME conversion efficiencies, irrespective of reaction time. Those FA to FAME conversion efficiencies occurred routinely at $\geq 90 \%$ ranging from $92.0 \pm 2.1$ to $97.0 \pm 0.2$. However, the FA to FAME conversion efficiencies seemed somewhat lower at a ratio of methanol to hexane of $1: 4$. This is presumably due to the lesser collision of methanolic solution with triglyceride as the concentration of methanol in reactant was low. ${ }^{\mathbf{4 1 , 4 2}}$ This result is similar to previously reported research that concluded an excess of co-solvent could decrease biodiesel yield. ${ }^{\mathbf{4 3 4 4}}$ The highest FA to FAME conversion efficiency occurred after $30 \mathrm{~min}$ in the simultaneous extractiontransesterification process using a ratio of methanol to hexane of $1: 2$.

The ratio of hexane to methanol had a significant effect on the extraction of $\mathrm{C}_{18: 2}$ and $\mathrm{C}_{18: 1}$ as shown in Fig. 4 . The extraction of $\mathrm{C}_{18: 2}$ was slightly lower at the ratio of hexane to methanol of $1: 4$ of the reaction time, achieving FA extraction efficiency range of $13.3 \pm 4.8$ to $23.3 \pm 18 \mathrm{~mol} \%$. In contrast, at the same ratio of hexane to methanol the extraction efficiency of $\mathrm{C}_{18: 1}$ was the highest of all the ratios. The average FA extraction efficiency in this study was $56.7 \mathrm{~mol} \%$, ranging from 52.7 to $78.1 \mathrm{~mol} \%$.

\subsection{Effect of catalyst reusability}

In order to reduce the total biodiesel cost, it is necessary to recycle the catalyst used..$^{45}$ Heterogeneous catalysts, which have different phases with triglycerides and methanol, could catalyze transesterification reactions over several times without losing their catalytic activity. Liu et al. $^{\mathbf{4 6}}$ demonstrated that heterogeneous $\mathrm{CaO}$ produced a high biodiesel yield after 20 times in the reaction cycle. The different phases between the catalyst and reactant allow simple separation for recycling the catalyst. However, this is almost impossible with a homogeneous catalyst which dissolves well in the reactant (methanol). Evaporation of the methanol phase containing sodium hydroxide, followed by addition of an appropriate volume of methanol (to ensure the amount of methanol used was constant) was conducted by Britton and Raston $^{47}$ to study recyclability of

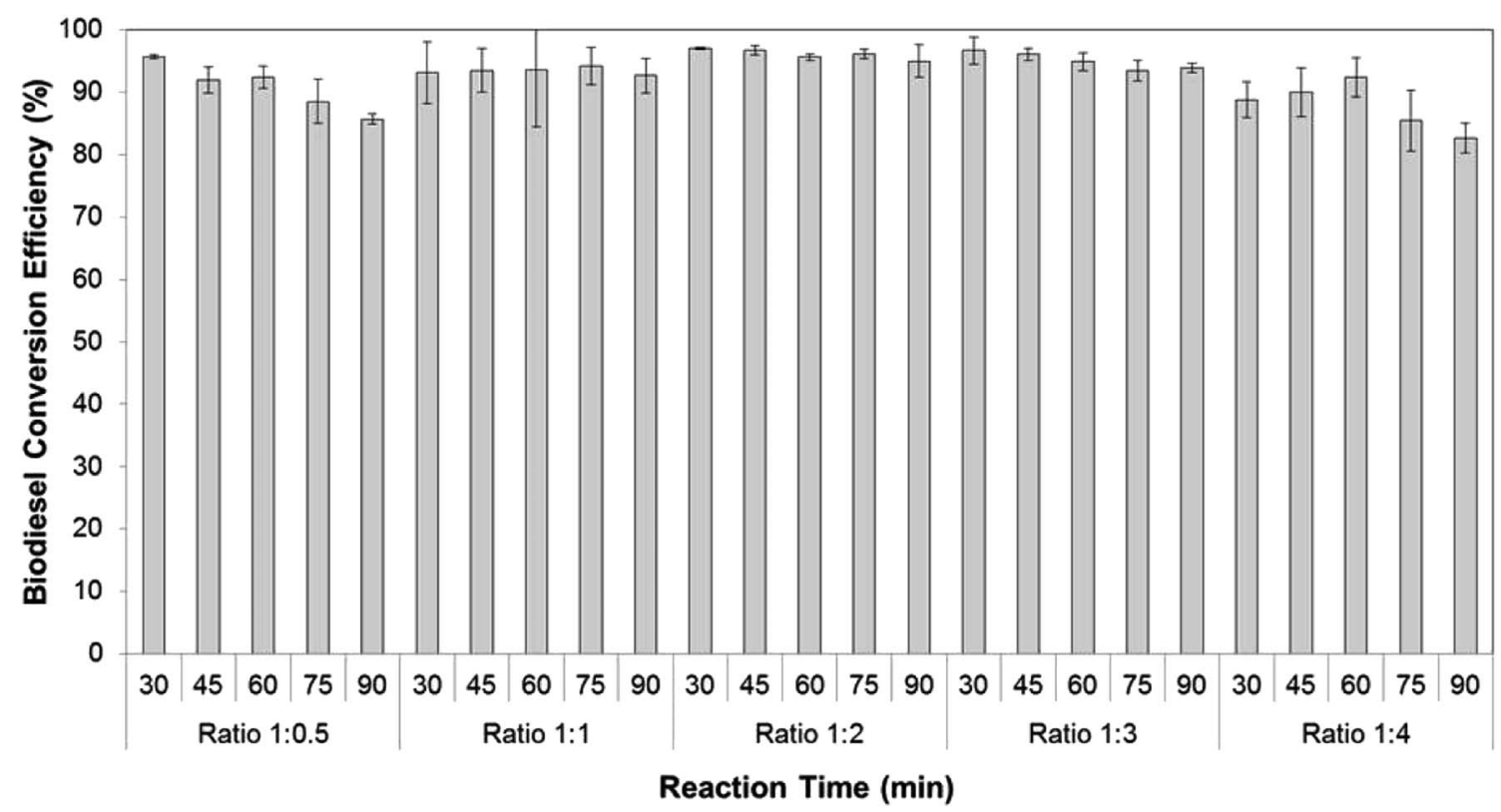

Fig. 3 The effect of ratio hexane to methanol on FA to FAME conversion efficiency with different reaction times. 


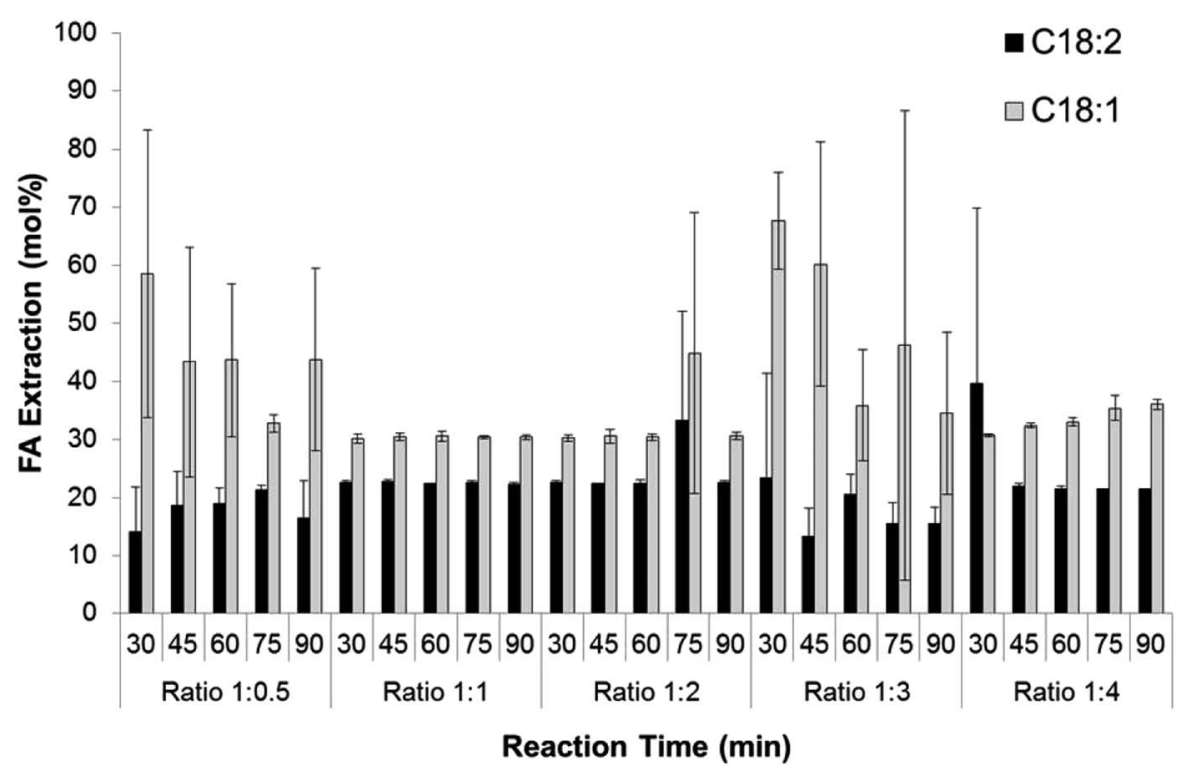

Fig. 4 The effect of ratio hexane to methanol on $\mathrm{C}_{18: 2}$ and $\mathrm{C}_{18: 1}$ extraction efficiency in different reaction times.

homogeneous base catalyst in vortex fluidic devices in mediated biodiesel production from sunflower oil. The biodiesel yield slightly decreased after the catalyst was used 3 times and declined to $<40 \%$ in further repeats. ${ }^{47}$ In contrast, in this study the biodiesel conversion efficiencies remained high $(\geq 90 \%)$ after 5 cycles. The parameters were set to $30 \mathrm{~min}$ of reaction time, catalyst concentration of $0.75 \mathrm{M}$ and ratio methanol to hexane of $1: 1$ to determine the effect of catalyst reusability while both the wet SCG biomass and hexane were replaced in every cycle. The methanolic solution was used directly without any additional treatment. After 5 repeats the biodiesel conversion efficiency only decreased by $5.8 \%$ from the $96.3 \% \pm 0.4$ observed in the first cycle. The constant catalytic activity is due to the concentration of sodium hydroxide in methanol remaining sufficiently high to catalyse transesterification reaction. The biodiesel conversion efficiency slightly decreased as the amount of glycerol increased which reduced the catalyst concentration.

The extraction efficiencies of $\mathrm{C}_{18: 2}$ and $\mathrm{C}_{18: 1}$ remained quite similar after 5 times reuse of the catalyst. The extraction efficiency of $\mathrm{C}_{18: 2}$ was in the range of 22.8 to $23 \mathrm{~mol} \%$ while $\mathrm{C}_{18: 1}$ was 29.5 to $34.9 \mathrm{~mol} \%$. These results were predicted as extraction and transesterification reaction proceeded in different chambers with no contact between catalyst and the lipids contained in wet SCG causing the catalyst to have no effect on FA extraction efficiency.

\subsection{Electricity and time consumption}

Electricity consumption of $i n$ situ transesterification of wet SCG using the RES method based on lab-scale was calculated roughly and compared with other two-step and one-step methods using either dry or wet SCG biomass (Table 2). The device used to conduct RES method was a regular hotplate magnetic stirrer which operates at a temperature of $65^{\circ} \mathrm{C}$ for 0.5 hours. The energy requirement to process 10 gram of wet SCG was $30 \mathrm{~kW} \mathrm{~h} \mathrm{~kg}^{-1}$, which is equivalent to $108 \mathrm{MJ} \mathrm{kg}^{-1}$ of wet biomass. The energy consumption for in situ transesterification of wet SCG using RES method was lower than in other published reports. ${ }^{17,21,36}$ In comparison to other in situ transesterifications of wet SCG, the RES method showed an energy saving of more than $99 \%$, as the other methods used a heating bath which

Table 2 Comparison energy consumption $\left(\mathrm{kW} \mathrm{h} \mathrm{kg}^{-1}\right)$ of biodiesel production from SCG based on this study, ref. 17, 21 and $36^{a}$

\begin{tabular}{|c|c|c|c|c|}
\hline Process & Two-step & One step & One step reflux & $\begin{array}{l}\text { One step reactive } \\
\text { extraction }\end{array}$ \\
\hline Drying & 7.5 & 7.5 & & \\
\hline Lipid extraction & 34.72 & - & & \\
\hline Transesterification & 6.4 & 60 & 3333.33 & 30 \\
\hline
\end{tabular}

${ }^{a}$ Average electricity cost in Indonesia is AUS\$ 9.24c per $\mathrm{kW} \mathrm{h.49}$ 
required $1 \mathrm{~kW} \mathrm{~h}$ operating for 2 hours to process 0.6 gram biomass. ${ }^{21}$ Furthermore, comparison with other in situ transesterification methods which used dry biomass showed that the RES method is not only more energy-wise, saving 56\% of energy, but also time-wise, as it eliminated the drying process which requires three days of sun drying and overnight drying at $105{ }^{\circ} \mathrm{C}$ using a hot air oven. ${ }^{17}$ The processing time and energy requirement of in situ transesterification of wet SCG using RES method was also lower compared to the two-step method which requires a separate extraction and transesterification process. The extraction process consumed $34.75 \mathrm{~kW} \mathrm{~h} \mathrm{~kg}^{-1}$ when using regular extractor apparatus. ${ }^{48}$ The RES method saved energy and time at $38 \%$ and $96.3 \%$, respectively. ${ }^{36}$

\section{Conclusion}

This study developed a new approach to the RES method of simultaneously extracting and converting lipid from wet SCG biomass to biodiesel in a single-step process at a mild reaction temperature and short reaction time. The FA to FAME conversion efficiency routinely produced more than $90 \%$ using sodium hydroxide in methanol as catalyst at a concentration of $0.75 \mathrm{M}$ and hexane as co-solvent. Interestingly, water contained in the wet SCG biomass did not affect biodiesel production. There was no significant effect of all the parameters studied except for the ratio of hexane to methanol. The FA extraction efficiencies occurred on average of $58.1 \mathrm{~mol} \%$ ranging from 48.6-78.1 mol\%. The electricity consumption assessment showed that the RES method could save 38-99\% of energy compared to other dry and wet two- and one-step biodiesel production processes. Together our studies provide important insights into a new strategy to utilize wet SCG biomass for biodiesel production before being discarded into the environment.

\section{Conflicts of interest}

There are no conflicts to declare.

\section{Acknowledgements}

The authors wish to thank the Ministry of Research, Technology and Higher Degree of the Republic of Indonesia through Universitas Sumatera Utara for research grant no. 11/E1/KPPTNBH/2019 date $29^{\text {th }}$ March 2019.

\section{References}

1 J. C. Escobar, E. S. Lora, O. J. Venturini, E. E. Yáñez, E. F. Castillo and O. Almazan, Renewable Sustainable Energy Rev., 2009, 13, 1275-1287.

2 R. Xu and Y. Mi, J. Am. Oil Chem. Soc., 2011, 88, 91-99.

3 S. H. Y. S. Abdullah, N. H. M. Hanapi, A. Azid, R. Umar, H. Juahir, H. Khatoon and A. Endut, Renewable Sustainable Energy Rev., 2017, 70, 1040-1051.

4 M. J. Haas, A. J. McAloon, W. C. Yee and T. A. Foglia, Bioresour. Technol., 2006, 97, 671-678.
5 A. W. Go, S. Sutanto, L. K. Ong, P. L. Tran-Nguyen, S. Ismadji and Y.-H. Ju, Renewable Sustainable Energy Rev., 2016, 60, 284-305.

6 E. K. Sitepu, K. Corbin, X. Luo, S. J. Pye, Y. Tang, S. C. Leterme, K. Heimann, C. L. Raston and W. Zhang, Bioresour. Technol., 2018, 266, 488-497.

7 F. H. Kasim and A. P. Harvey, Chem. Eng. J., 2011, 171, 13731378.

8 S. Kaul, J. Porwal and M. O. Garg, J. Am. Oil Chem. Soc., 2010, 87, 903-908.

9 A. A. Koutsouki, E. Tegou, S. Kontakos, M. G. Kontominas, P. J. Pomonis and G. Manos, Fuel Process. Technol., 2015, 134, 122-129.

10 E. K. Sitepu, D. B. Jones, Y. Tang, S. C. Leterme, K. Heimann, W. Zhang and C. L. Raston, ChemComm, 2018, 54, 1208512088.

11 E. K. Sitepu, D. B. Jones, Z. Zhang, Y. Tang, S. C. Leterme, K. Heimann, C. L. Raston and W. Zhang, Bioresour. Technol., 2019, 273, 431-438.

12 S. R. Dasari, A. J. Chaudhary, V. V. Goud, N. Sahoo and V. N. Kulkarni, Energy Convers. Manage., 2017, 142, 200-214. 13 R. A. Sheldon, ACS Sustainable Chem. Eng., 2018, 6, 32-48.

14 R. Dominguez-Faus, S. E. Powers, J. G. Burken and P. J. Alvarez, Environ. Sci. Technol., 2009, 43, 3005-3010.

15 M. Canakci, Bioresour. Technol., 2007, 98, 183-190.

16 E. C. G. Aguieiras, E. D. Cavalcanti-Oliveira and D. M. G. Freire, Fuel, 2015, 159, 52-67.

17 N. Tuntiwiwattanapun, E. Monono, D. Wiesenborn and C. Tongcumpou, Ind. Crops Prod., 2017, 102, 23-31.

18 N. Kondamudi, S. K. Mohapatra and M. Misra, J. Agric. Food Chem., 2008, 56, 11757-11760.

19 A. Arulrajah, F. Maghoolpilehrood, M. M. Disfani and S. Horpibulsuk, J. Cleaner Prod., 2014, 72, 181-186.

20 R. Campos-Vega, G. Loarca-Piña, H. A. Vergara-Castañeda and B. D. Oomah, Trends Food Sci. Technol., 2015, 45, 24-36.

21 J. Park, B. Kim and J. W. Lee, Bioresour. Technol., 2016, 221, 55-60.

22 R. Cruz, M. M. Cardoso, L. Fernandes, M. Oliveira, E. Mendes, P. Baptista, S. Morais and S. Casal, J. Agric. Food Chem., 2012, 60, 7777-7784.

23 I. C. Organization, New coffee consumption table, 2017.

24 S. K. Karmee, Waste Manag., 2018, 72, 240-254.

25 Y. Liu, Q. Tu, G. Knothe and M. Lu, Fuel, 2017, 199, 157-161. 26 N. Tuntiwiwattanapun and C. Tongcumpou, Ind. Crops Prod., 2018, 117, 359-365.

27 K. Sander and G. Murthy, Int. J. Life Cycle Assess., 2010, 15, 704-714.

28 J. Park, B. Kim, J. Son and J. W. Lee, Bioresour. Technol., 2018, 249, 494-500.

29 J. Son, B. Kim, J. Park, J. Yang and J. W. Lee, Bioresour. Technol., 2018, 259, 465-468.

30 P. Hidalgo, C. Toro, G. Ciudad, S. Schober, M. Mittelbach and R. Navia, Energy Fuels, 2014, 28, 3814-3820.

31 P. Hidalgo, G. Ciudad, S. Schober, M. Mittelbach and R. Navia, Bioresour. Technol., 2015, 181, 32-39.

32 S. R. Dasari and V. V. Goud, Process Saf. Environ. Prot., 2017, 107, 373-387. 
33 J. Folch, M. Lees and G. H. Sloane Stanley, J. Biol. Chem., 1957, 226, 497-509.

34 M. Tariq, S. Ali, F. Ahmad, M. Ahmad, M. Zafar, N. Khalid and M. A. Khan, Fuel Process. Technol., 2011, 92, 336-341.

35 G. Knothe and J. A. Kenar, Eur. J. Lipid Sci. Technol., 2004, 106, 88-96.

36 Z. Al-Hamamre, S. Foerster, F. Hartmann, M. Kröger and M. Kaltschmitt, Fuel, 2012, 96, 70-76.

37 B. B. Uzun, M. Kılıç, N. Özbay, A. E. Pütün and E. Pütün, Energy, 2012, 44, 347-351.

38 S. Nasreen, M. Nafees, L. A. Qureshi, M. S. Asad, A. Sadiq and S. D. Ali, in Biofuels-State of Development, IntechOpen, 2018. 39 F. Ma and M. A. Hanna, Bioresour. Technol., 1999, 70, 1-15.

40 H. C. Nguyen, S.-H. Liang, S.-Y. Li, C.-H. Su, C.-C. Chien, Y.-J. Chen and D. T. M. Huong, J. Taiwan Inst. Chem. Eng., 2018, 85, 165-169.

41 L. T. Thanh, K. Okitsu, Y. Sadanaga, N. Takenaka, Y. Maeda and H. Bandow, Fuel, 2013, 103, 742-748.
42 Y. Zhang, Y. Li, X. Zhang and T. Tan, Bioresour. Technol., 2015, 196, 712-715.

43 Y. Alhassan, N. Kumar, I. M. Bugaje, H. S. Pali and P. Kathkar, Energy Convers. Manage., 2014, 84, 640-648.

44 J. M. Encinar, A. Pardal and N. Sánchez, Fuel, 2016, 166, 5158.

45 M. R. Avhad and J. M. Marchetti, Renewable Sustainable Energy Rev., 2015, 50, 696-718.

46 X. Liu, H. He, Y. Wang, S. Zhu and X. Piao, Fuel, 2008, 87, 216-221.

47 J. Britton and C. L. Raston, RSC Adv., 2014, 4, 49850-49854.

48 J. Chopra, R. Dineshkumar, M. Bhaumik, G. Dhanarajan, R. Kumar and R. Sen, RSC Adv., 2016, 6, 70364-70373.

49 S. Epifany, Indonesia Electricity Tariff Still Competitive in ASEAN Region, https://www.infrastructureasiaonline.com/ government/indonesia-electricity-tariff-still-competitiveasean-region, accessed 12 February 2019. 